\title{
An Accurate Closed-Form Approximation of the Distributed MIMO Outage Probability
}

\author{
Fabien Héliot, Member, IEEE, Reza Hoshyar, Member, IEEE, and Rahim Tafazolli, Member, IEEE,
}

\begin{abstract}
The mutual information (MI) of multiple-input multiple-output (MIMO) system over Rayleigh fading channel is known to asymptotically follow a normal probability distribution. In this paper, we first prove that the MI of distributed MIMO (DMIMO) system is also asymptotically equivalent to a Gaussian random variable $(\mathrm{RV})$ by deriving its moment generating function (MGF) and by showing its equivalence with the MGF of a Gaussian RV. We then derive an accurate closed-form approximation of the outage probability for DMIMO system by using the mean and variance of the MI and show the uniqueness of its formulation. Finally, several applications for our analysis are presented.
\end{abstract}

Index Terms-Cooperative communication, distributed MIMO, asymptotical analysis, outage probability.

\section{INTRODUCTION}

Calculation of the channel capacity for multiple-input multiple-output (MIMO) system over the Rayleigh fading channel has attracted considerable research interests in the past decades [1]-[3]. Recently, it has been shown by various authors using different methods that the mutual information (MI), also known as instantaneous capacity, of the MIMO Rayleigh fading channel is equivalent to a Gaussian random variable (RV) [4]-[7]. This result has been theoretically obtained for a large number of inputs and outputs of the MIMO channel and has been confirmed by simulations for even a small number of inputs and outputs [5], [6], [8]. Moreover, this result has been used to obtain an approximation of the outage probability for MIMO system in [7]. As far as distributed MIMO (DMIMO) system is concerned, its MI has a similar formulation as the MI of MIMO system over the semicorrelated Rayleigh fading channel when the system model for DMIMO in [9] or [10] is assumed. An exact computable formula of the characteristic function has been derived for this type of channel in [11] and the equivalence of the MI with a Gaussian RV has been suggested for a large number of antennas but has only been shown numerically. Then in [10], the equivalence has been stated and closed-form approximations of the mean and variance of the MI have been derived at high signal-to-noise ratios (SNRs) for DMIMO systems having a large number of nodes and antennas.

Based on the system model presented in Section II [9], [10], we first prove in Section III that the MI of a $m$-nodes DMIMO system is asymptotically equivalent to a Gaussian

F. Héliot, R. Hoshyar, and R. Tafazolli are with the Centre for Communication Systems Research, Faculty of Electronics \& Physical Sciences, University of Surrey, Guildford, GU2 7XH, UK (phone: +44 1483689 492; fax: +44 1483 686 011; E-mail: F.Heliot@Surrey.ac.uk). This work has been performed in the framework of the FP7 project ROCKET IST-215282 STP, which is funded by the European Commission.
RV by using random matrix theory [12] and the multiple saddle point integration technique [13]. We derive the moment generating function (MGF) of the MI for a large number of nodes and antennas, and we show that its formulation is the same as the MGF of a Gaussian RV. We obtain the moments of the MI by identifying them with the moments of a Gaussian RV. Next, we derive a closed-form approximation of the outage probability for DMIMO system, which becomes tighter as the number of nodes and antennas of the system increases, by using the mean and variance of the MI. Our mean and variance expressions are given in terms of the roots of a polynomial of degree $m+1$. In Section IV, we first prove that the root selection is unique for $m=1$, we then generalize the proof for any $m$ values and, hence, demonstrate the uniqueness of our closed-form approximation. Accuracy of our approximation is discussed in Section V and is shown for even a small number of nodes and antennas. Several applications for our analysis are presented in Section $\mathrm{V}$ and, finally, conclusions are drawn in Section VI.

In comparison with the work in [11], we prove the Gaussian behavior of the MI and provide an accurate closed-form approximation of the MI MGF that can be used for any configuration of rows and columns of the channel matrix. Whereas the case where the number of rows is greater than the number of columns has not been tackled by the analysis in [11]. In addition, our approximation can be used for both DMIMO downlink and uplink scenarios and for any SNR settings. Whereas the approximation in [10] has been designed for high SNR values only. Some preliminary results of this work have been presented in [14].

\section{SyStem MODEL}

We consider a DMIMO communication system composed of several nodes, which are in different locations, where $m$ base stations (BSs) equipped of $p$ antennas cooperate to transmit/ receive data to/from a mobile station (MS) equipped with $q$ antennas, as it is illustrated in Fig. 1. We also assume as in [9] and [10] that all $\mathrm{mp}$ antennas have a separate feeder to the central unit where all signal processing is done. The matrices $\boldsymbol{\Sigma}_{i}$ and $\mathbf{H}_{i}$ represent the average path loss/shadowing and the MIMO Rayleigh fading channel, respectively, between the $i$ th BS and the MS, $i \in\{1, \ldots, m\}$. The equivalent channel model of the system depicted in Fig. 1 is then defined as $\widetilde{\mathbf{H}}=\boldsymbol{\Sigma} \odot \mathbf{H}$, where $\mathbf{H}=\left[\mathbf{H}_{1}^{\dagger}, \mathbf{H}_{2}^{\dagger}, \ldots, \mathbf{H}_{m}^{\dagger}\right]^{\dagger},(.)^{\dagger}$ is the Hermitian transpose operator, $\odot$ is the entry-wise product between any two matrices, $\widetilde{\mathbf{H}} \in \mathbb{C}^{N_{r} \times N_{t}}, \mathbf{H} \in \mathbb{C}^{N_{r} \times N_{t}}$ and $\boldsymbol{\Sigma} \in \mathbb{R}_{+}^{N_{r} \times N_{t}}$ with $\mathbb{R}_{+}=\{x \in \mathbb{R} \mid x \geq 0\}$. Moreover, 


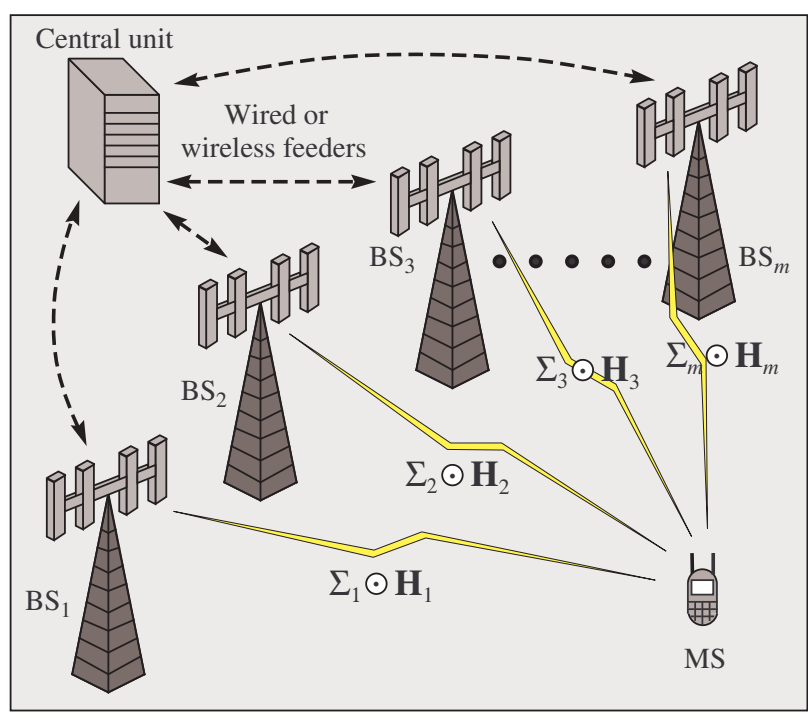

Fig. 1. Distributed MIMO system model.

$\boldsymbol{\Sigma}=\boldsymbol{\Gamma} \triangleq\left[\boldsymbol{\Gamma}_{1}^{\dagger}, \boldsymbol{\Gamma}_{2}^{\dagger}, \ldots, \boldsymbol{\Gamma}_{m}^{\dagger}\right]^{\dagger}$ and $\boldsymbol{\Sigma}=\boldsymbol{\Gamma}^{\dagger}$ in the uplink and downlink cases, respectively, with $\Gamma_{i} \in \mathbb{R}_{+}^{p \times q}$. Furthermore, the total number of transmit and receive antennas of the DMIMO system is defined as $N_{t}$ and $N_{r}$, respectively. In the uplink case $N_{t}=n=q$ and $N_{r}=m p$, whereas in the downlink case $N_{t}=m p, N_{r}=q$ and $n=p$, where $n$ is the number of transmit antenna per node. Accordingly, the receive signal $\mathbf{r} \in \mathbb{C}^{N_{r} \times 1}$ can be expressed as

$$
\mathbf{r}=\widetilde{\mathbf{H}} \mathbf{s}+\mathbf{n},
$$

where $\mathbf{s} \in \mathbb{C}^{N_{t} \times 1}$ is the transmit signal with average transmit power $P$ and $\mathbf{n} \in \mathbb{C}^{N_{r} \times 1}$ is the noise vector containing the random noise samples from each receiver with average noise power $N$. We assume that $\mathbf{H}$ is a random matrix having independent and identically distributed (i.i.d.) complex circular Gaussian entries with zero-mean and unit variance. The MI of the composite channel $\widetilde{\mathbf{H}}, C(\widetilde{\mathbf{H}})$, can then be defined as

$$
C(\widetilde{\mathbf{H}}) \triangleq \lambda \ln \left|\mathbf{I}_{N_{r}}+\frac{\gamma}{n} \widetilde{\mathbf{H}} \widetilde{\mathbf{H}}^{\dagger}\right|,
$$

where $\gamma \triangleq P / N, \gamma \in \mathbb{R}_{+}$, is the average SNR, $\mathbf{I}_{N_{r}}$ is a $N_{r} \times$ $N_{r}$ identity matrix and $\lambda=1$ if the capacity is expressed in nats/s/Hz or $\lambda=\frac{1}{\ln (2)}$ if the capacity is expressed in bits/s/Hz. Notice that if $p=1$ and $\boldsymbol{\Gamma}_{i}=\sqrt{\gamma_{i}} \mathbf{1}^{1 \times q}, \gamma_{i} \in \mathbb{R}^{+}$, where $\mathbf{1}^{1 \times q}$ is a $1 \times q$ vector with all its elements equal to one, then (2) is the expression of the MIMO semicorrelated Rayleigh fading MI [11].

\section{Closed-form APproximation of the DMimo OUTAGE PROBABILITY}

Following a similar approach as in [4] and [5], we first prove that $C(\widetilde{\mathbf{H}})$ is asymptotically equivalent to a Gaussian RV and then derive its mean and variance for large values of $m, p$ and $q$.
The MGF of the RV $C(\widetilde{\mathbf{H}})$ can be expressed as $M_{C(\widetilde{\mathbf{H}})}(-u / \lambda) \triangleq \mathrm{E}_{\mathbf{H}}\{\exp [(-u / \lambda) C(\widetilde{\mathbf{H}})]\}$

$$
\begin{aligned}
& =\pi^{-N_{r} N_{t}} \int_{\mathbb{C}^{N_{r} \times N_{t}}} e^{\left(-\|\mathbf{H}\|_{F}^{2}\right)} \phi(\widetilde{\mathbf{H}})^{-u} d \mathbf{H}, \\
& =\pi^{-N_{r} N_{t}} \int_{\mathbb{C}^{N_{r} \times N_{t}}} \int_{\mathbb{C}^{N_{r} \times u}} \int_{\mathbb{C}^{N_{t} \times u}} e^{-\pi \operatorname{tr}\left[\left(\mathbf{X}^{\dagger} \mathbf{X}+\mathbf{Y}^{\dagger} \mathbf{Y}\right)\right]} \psi( \\
& \times d \mathbf{H} d \mathbf{X} d \mathbf{Y}
\end{aligned}
$$

where $\mathrm{E}_{\mathbf{H}}\{$.$\} is the expectation over \mathbf{H},\|\cdot\|_{F}$ denotes the Frobenius norm, $\operatorname{tr}[$.$] is the trace of a matrix$ and $\phi(\widetilde{\mathbf{H}})=\left|\mathbf{I}_{N_{r}}+\frac{\gamma}{n} \widetilde{\mathbf{H}} \widetilde{\mathbf{H}}^{\dagger}\right|$. In addition, $\psi(\mathbf{H})=$ $\exp \left(-\operatorname{tr}\left[j\left(\widetilde{\mathbf{H}} \mathbf{B}^{\dagger}+\mathbf{B} \widetilde{\mathbf{H}}^{\dagger}\right)+\mathbf{H} \mathbf{H}^{\dagger}\right]\right), \mathbf{B}=\pi \sqrt{\gamma / n} \mathbf{X} \mathbf{Y}^{\dagger}$, $\mathbf{B} \in \mathbb{C}^{N_{r} \times N_{t}}, \mathbf{X} \in \mathbb{C}^{N_{r} \times u}, \mathbf{Y} \in \mathbb{C}^{N_{t} \times u}$ and $u$ is a dummy variable used in the replica method [15]. By integrating $\psi(\mathbf{H})$ with respect to $\mathbf{H}$, (3) can be rewritten as

$$
\begin{aligned}
M_{C(\widetilde{\mathbf{H}})}(-u / \lambda) & =\int_{\mathbb{C}^{N_{r} \times u}} \int_{\mathbb{C}^{N_{t} \times u}} e^{-\pi \operatorname{tr}\left[\left(\mathbf{X}^{\dagger} \mathbf{X}+\mathbf{Y}^{\dagger} \mathbf{Y}\right)\right]} \\
& \times e^{-\pi^{2} \frac{\gamma}{n} \operatorname{tr}\left[\left(\boldsymbol{\Sigma} \odot \mathbf{X} \mathbf{Y}^{\dagger}\right)\left(\boldsymbol{\Sigma} \odot \mathbf{X} \mathbf{Y}^{\dagger}\right)^{\dagger}\right]} d \mathbf{X} d \mathbf{Y} .
\end{aligned}
$$

Let $\boldsymbol{\Gamma}_{i}=\sqrt{\gamma_{i}} \mathbf{1}^{p \times v}$, then the following equalities $(\boldsymbol{\Gamma} \odot$ $\left.\mathbf{X} \mathbf{Y}^{\dagger}\right)=(\boldsymbol{\Gamma} \odot \mathbf{X}) \mathbf{Y}^{\dagger}$ and $\left(\boldsymbol{\Gamma}^{\dagger} \odot \mathbf{X} \mathbf{Y}^{\dagger}\right)=\mathbf{X}\left(\boldsymbol{\Gamma}^{\dagger} \odot \mathbf{Y}^{\dagger}\right)$ hold in the uplink and downlink, respectively, with $v=q$ in the left hand side and $v=u$ in the right hand side of each equality. Thus, (4) can be re-expressed in the uplink as

$$
\begin{aligned}
M_{C(\tilde{\mathbf{H}})}(-u / \lambda) & =\int_{\mathbb{C}^{N_{r} \times u}} \int_{\mathbb{C}^{N_{t} \times u}} e^{-\pi \operatorname{tr}\left[\left(\mathbf{X}^{\dagger} \mathbf{X}+\mathbf{Y}^{\dagger} \mathbf{Y}\right)\right]} \\
& \times e^{-\pi^{2} \frac{\gamma}{n} \operatorname{tr}\left[\mathbf{W} \mathbf{Y}^{\dagger} \mathbf{Y} \mathbf{W}^{\dagger}\right]} d \mathbf{X} d \mathbf{Y},
\end{aligned}
$$

where $\mathbf{W} \triangleq(\boldsymbol{\Gamma} \odot \mathbf{X})$. Following some simplifications, (5) is modified as

$$
\begin{aligned}
M_{C(\tilde{\mathbf{H}})}(-u / \lambda) & =\exp \left(u n\left[(m \alpha+\beta) \ln (\omega)+\alpha \sum_{i=1}^{m} \ln \left(v_{i}\right)\right]\right) \\
& \times\left(\frac{n}{j 2 \pi}\right)^{u^{2}} \int_{\mathcal{D}_{d}^{j}} \int_{\mathcal{D}_{g}} \exp (n \varphi(\mathbf{D}, \mathbf{G})) d \mathbf{D} d \mathbf{G},
\end{aligned}
$$

where $\alpha \triangleq p / n, \beta \triangleq q / n, v_{i} \triangleq 1 / \gamma_{i}$ and $\omega \triangleq$ $1 / \sqrt{\gamma}$. In addition, $\mathcal{D}_{d}^{j} \triangleq \mathbf{D}_{0}+\left(j \mathbb{R}^{u \times u}\right), \mathcal{D}_{g} \triangleq \mathbf{G}_{0}+$ $\left(\mathbb{R}^{u \times u}\right), \mathbf{D}, \mathbf{G}, \mathbf{D}_{0}, \mathbf{G}_{0} \in \mathbb{C}^{u \times u}$ and $\varphi(\mathbf{D}, \mathbf{G}) \triangleq \operatorname{tr}(\mathbf{D G})-$ $\alpha \sum_{i=1}^{m} \ln \left|\omega v_{i} \mathbf{I}_{u}+\mathbf{D}\right|-\beta \ln \left|\omega \mathbf{I}_{u}+\mathbf{G}\right|$. At this stage, we apply the multidimensional saddle point integration method in [13] for integrating the integral part of (6). After further derivation steps and simplifications, we obtain

$$
\begin{aligned}
& M_{C(\widetilde{\mathbf{H}})}(-u / \lambda) \rightarrow \exp \left(u n \left[\beta \ln \left(\frac{\omega d_{0}}{\beta}\right)+\alpha \sum_{i=1}^{m} \frac{d_{0}}{d_{0}+\omega v_{i}}\right.\right. \\
& \left.\left.+\alpha \sum_{i=1}^{m} \ln \left(\frac{\omega v_{i}}{d_{0}+\omega v_{i}}\right)\right]+\frac{u^{2}}{2}\left[-\ln \left(1-\widehat{d_{0}} \widehat{g_{0}}\right)\right]\right),
\end{aligned}
$$

where $\widehat{d_{0}}=\alpha \sum_{i=1}^{m}\left(d_{0}+\omega v_{i}\right)^{-2}, \widehat{g_{0}}=d_{0}^{2} / \beta$ and $d_{0}$ is one of the $m+1$ roots of the following degree- $m$ polynomial

$P_{m}(d)=(d \omega-\beta) \prod_{i=1}^{m}\left(d+\omega v_{i}\right)+d \alpha \sum_{i=1}^{m}\left[\prod_{\substack{k=1 \\ k \neq i}}^{m}\left(d+\omega v_{k}\right)\right]$. 
Knowing that the MGF of any Gaussian RV $Z$ is $M_{Z}(t) \triangleq$ $\mathrm{E}_{Z}\{\exp (t Z)\}=\exp \left(t \mu_{z}+\frac{t^{2}}{2} \sigma_{z}^{2}\right)$, with $\mu_{z}$ and $\sigma_{z}^{2}$ being the mean and variance of $Z$, respectively, we conclude by matching (7) with $M_{Z}(t)$ that $C(\mathbf{H})$ is asymptotically equivalent to a Gaussian RV with respective mean and variance given by

$$
\left\{\begin{aligned}
\mu_{C(\widetilde{\mathbf{H}})} & =-\lambda n\left[\alpha\left\{m-\sum_{i=1}^{m}\left[\ln \left(\frac{d_{0}+\omega v_{i}}{\omega v_{i}}\right)+\frac{\omega v_{i}}{d_{0}+\omega v_{i}}\right]\right\}\right. \\
& \left.+\beta \ln \left(\frac{\omega d_{0}}{\beta}\right)\right] \\
\sigma_{C(\widetilde{\mathbf{H}})}^{2} & =-\lambda^{2} \ln \left(1-\frac{\alpha}{\beta} \sum_{i=1}^{m}\left(\frac{d_{0}}{d_{0}+\omega v_{i}}\right)^{2}\right) .
\end{aligned}\right.
$$

Notice that the expressions of $\mu_{C(\widetilde{\mathbf{H}})}$ and $\sigma_{C(\widetilde{\mathbf{H}})}^{2}$ in (9) can be used for both uplink and downlink scenarios with $n=q, \alpha=$ $p / q, \beta=1$ and $n=p, \alpha=1, \beta=q / p$, respectively.

The probability that the transmission rate $R$ exceeds the MI of the channel $C(\widetilde{\mathbf{H}})$ is defined as $P_{\text {out }} \triangleq \mathrm{P}(C(\widetilde{\mathbf{H}})<R)$ when assuming that $\widetilde{\mathbf{H}}$ is chosen randomly and kept constant over a codeword transmission [7]. Hence, the outage probability of DMIMO system is well approximated by

$$
P_{\text {out }} \approx Q\left(\left[\mu_{C(\tilde{\mathbf{H}})}-R\right] / \sigma_{C(\widetilde{\mathbf{H}})}\right)
$$

for large values of $p, q$ and $m>1$, since $C(\widetilde{\mathbf{H}})$ is equivalent to a Gaussian RV in this case.

\section{UNiQUENESS OF THE SELECTION OF $d_{0}$}

The mean and variance of $C(\widetilde{\mathbf{H}})$ in (9) are both expressed in terms of $d_{0}$. The root $d_{0}$ can be selected amongst the $m+1$ roots of $P_{m}(d)$, and hence $P_{\text {out }}$ in (10) would have a unique expression only if the selection of $d_{0}$ is unique for any given $m \geq 2$. The mean $\mu_{C(\widetilde{\mathbf{H}})}$ and variance $\sigma_{C(\widetilde{\mathbf{H}})}^{2}$ are constrained such that $\mu_{C(\widetilde{\mathbf{H}})} \in \mathbb{R}$ and $\sigma_{C(\tilde{\mathbf{H}})}^{2} \in \mathbb{R}_{+}^{\star}$ with $\mathbb{R}_{+}^{\star}=\left\{x \in \mathbb{R}_{+} \mid x \neq 0\right\}$. Applying $\sigma_{C(\tilde{\mathbf{H}})}^{2} \in \mathbb{R}_{+}^{\star}$ in (9), we get [16]

$$
\begin{gathered}
\sigma_{C(\tilde{\mathbf{H}})}^{2} \in \mathbb{R}_{+}^{*} \Leftrightarrow 0<\sum_{i=1}^{m}\left(\frac{d_{0}}{d_{0}+\omega v_{i}}\right)^{2}<\frac{q}{p} \Rightarrow \\
\begin{cases}d_{0} \in\left(-\infty, d_{\mathrm{b}}\right) \cup\left(d_{\mathrm{a}},+\infty\right) & , \text { if } q>p m, \\
d_{0} \in\left(d_{\mathrm{b}}, d_{\mathrm{a}}\right) & , \text { if } q<p m, \\
d_{0}>-\omega \widehat{v} / 2 & , \text { if } q=p m,\end{cases}
\end{gathered}
$$

where $\widehat{v}=\max _{i}\left\{v_{i}\right\}$ for $i \in\{1, m\}, d_{\mathrm{a}}=$ $\omega \widehat{v}\left[\frac{q}{p m-q}+\frac{\sqrt{p m q}}{|p m-q|}\right]$, and $d_{\mathrm{b}}=\omega \widehat{v}\left[\frac{q}{p m-q}-\frac{\sqrt{p m q}}{|p m-q|}\right]$. Clearly, $d_{\mathrm{b}} \leq 0$ if $q \neq p m, d_{\mathrm{a}} \geq 0$ only if $q<p m$ and the imaginary part of $d_{0}$ is null. In addition, we simply obtain

$$
\mu_{C(\widetilde{\mathbf{H}})} \in \mathbb{R} \Leftrightarrow \ln \left(\omega d_{0} / \beta\right) \in \mathbb{R} \Leftrightarrow d_{0} \geq 0 .
$$

by applying $\mu_{C(\widetilde{\mathbf{H}})} \in \mathbb{R}$ in (9). Finally, using the results in (11) and (12), $d_{0}$ is such that

$$
d_{0} \in \mathbb{R}_{+} \text {if } q \geq p m, \text { or } d_{0} \in\left[0, d_{\mathrm{a}}\right) \text { if } q<p m .
$$

Thus, $d_{0}$ should be appropriately selected to fulfill the constraint set in (13).

\section{A. MIMO case $(m=1)$ [7]}

In the case of $m=1$, the polynomial in (8) can be reexpressed as $P_{1}(d)=d^{2} \omega+d\left(\omega^{2} v_{1}+\alpha-\beta\right)-\beta \omega v_{1}$. Then, the two roots of $P_{1}(d)$ are directly obtained by solving the quadratic equation $P_{2}(d)=0$ such that

$$
d_{1, n}=\frac{-s+(-1)^{n} \sqrt{s^{2}+4 \beta \omega^{2} v_{1}}}{2 \omega},
$$

where $s=\left(\omega^{2} v_{1}+\alpha-\beta\right)$. Clearly, $d_{1,0} \geq 0$ and $d_{1,1} \leq 0$ for any $\omega, v_{1} \geq 0$ and $\alpha, \beta>0$. Therefore, the polynomial $P_{1}(d)$ has a unique real nonnegative root, i.e. $d_{1,0}$, which fulfils the constraint set in (13). Furthermore, the polynomial $P_{1}(d)$ is clearly such that

$$
P_{1}(d)\left\{\begin{array}{l}
\leq 0, \text { for } d \in\left[d_{1,1}, d_{1,0}\right], \\
\geq 0, \text { for } d \in\left(-\infty, d_{1,1}\right] \cup\left[d_{1,0},+\infty\right) .
\end{array}\right.
$$

Since $P_{1}\left(-\omega v_{1}\right)=-\alpha \omega v_{1} \leq 0$, hence, $-\omega v_{1} \in\left[d_{1,1}, d_{1,0}\right]$. Moreover we know that $-\omega v_{1} \leq 0$, it then implies that

$$
-\infty<d_{1,1} \leq-\omega v_{1} \leq 0 \leq d_{1,0}<+\infty .
$$

\section{B. DMIMO case $(m \geq 2)$}

From the definition of $P_{m}(d)$ in (8), we can express $P_{m}(d)$ as a sequence where the initial term is $P_{1}(d)$ and the general term is given by

$$
P_{m}(d)=\left(d+\omega v_{m}\right) P_{m-1}(d)+\alpha d \prod_{k=1}^{m-1}\left(d+\omega v_{k}\right),
$$

for $m \geq 2$. We denote $\Upsilon=\left[v_{1}, \ldots, v_{m}\right]$ and $\bar{\Upsilon}=$ $\left[\bar{v}_{1}, \ldots, \bar{v}_{m}\right]$, where $\bar{\Upsilon}$ is the ordered version of the vector $\Upsilon$ such that $0 \leq \bar{v}_{1} \leq \ldots \leq \bar{v}_{m}$, i.e. $\bar{v}_{1}=\min _{i}\{\Upsilon\}$ and $\bar{v}_{m}=\max _{i}\{\Upsilon\}$ for $i \in\{1, m\}$. The values of $P_{m}\left(d=-\omega \bar{v}_{i}\right)$ can be obtained by using (17), as follows, $P_{m}\left(-\omega \bar{v}_{i}\right)$

$$
=\alpha \omega^{m} \bar{v}_{i} \prod_{k=1}^{m-1}\left(\bar{v}_{i}-\bar{v}_{k}\right)\left\{\begin{array}{l}
=0, \text { if } \bar{v}_{i}=\bar{v}_{k}, i \in\{1, m\}, \\
>0, \text { if } \bar{v}_{i} \neq \bar{v}_{k}, i \in \mathbb{S}_{1}, \\
<0, \text { if } \bar{v}_{i} \neq \bar{v}_{k}, i \in \mathbb{S}_{2},
\end{array}\right.
$$

where the sets $\mathbb{S}_{1}$ and $\mathbb{S}_{2}$ are defined as $\mathbb{S}_{1}=\{m-2 l \mid l \in$ $\{0,\lfloor(m-1) / 2\rfloor\}\}$ and $\mathbb{S}_{2}=\{m-(2 l+1) \mid l \in\{0,\lfloor(m-$ $1) / 2\rfloor\}, i \neq 0\}$, respectively, and $\lfloor$.$\rfloor is the integral floor$ operator. Moreover, we consider that the polynomial $P_{m}(d)$ has $m+1$ roots denoted $d_{m, k}, k \in\{0, m\}$, which are ordered as follows $d_{m, m} \leq d_{m, m-1} \leq \ldots \leq d_{m, 0}$.

In the case that $m=2, \omega, \bar{v}_{1}, \bar{v}_{2}>0, \bar{v}_{1} \neq \bar{v}_{2}$ and $d_{1,0}>$ 0 , we obtain by evaluating $P_{2}(d)$ at $d=-\omega \bar{v}_{2}, d=-\omega \bar{v}_{1}$ in (18), and $d=0, d=d_{1,0}$ in (17), the following inequalities

$$
P_{2}\left(-\omega \bar{v}_{2}\right)>0, P_{2}\left(-\omega \bar{v}_{1}\right)<0, P_{2}(0)<0, P_{2}\left(d_{1,0}\right)>0 .
$$

Knowing that $\lim _{d \rightarrow-\infty} P_{2}(d)=-\infty,-\omega \bar{v}_{2}<-\omega \bar{v}_{1}$, and $-\omega \bar{v}_{1}<0<d_{1,0}^{d \rightarrow-\infty}$ according to (16), it implies that the roots $d_{2,2}, d_{2,1}$ and $d_{2,0}$ of $P_{2}(d)$ belong to the following intervals $\left(-\infty,-\omega \bar{v}_{2}\right),\left(-\omega \bar{v}_{2},-\omega \bar{v}_{1}\right)$ and $\left(0, d_{1,0}\right)$, respectively. As a result, the inequality

$-\infty<d_{2,2} \leq-\omega \bar{v}_{2} \leq d_{2,1} \leq-\omega \bar{v}_{1} \leq 0 \leq d_{2,0} \leq d_{1,0}<+\infty$ 


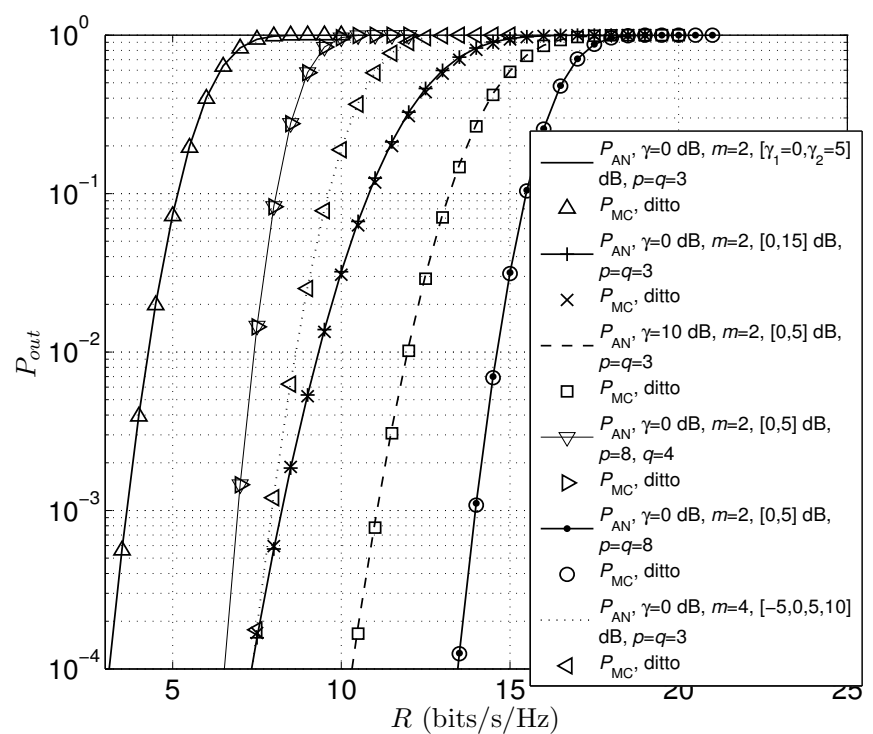

Fig. 2. Comparison of the outage probabilities $P_{\mathrm{AN}}$ and $P_{\mathrm{MC}}$, which have been obtained via (10) and MC simulation, respectively, as a function of the rate $R$ and for various SNR settings, numbers of nodes and transmit antennas.

holds when the conditions $\omega, \bar{v}_{1}, \bar{v}_{2}>0, d_{1,0}>0$ are relaxed to $\omega, \bar{v}_{1}, \bar{v}_{2} \geq 0, d_{1,0} \geq 0$. Hence, (20) indicates that, like $P_{1}(d), P_{2}(d)$ has a unique nonnegative root, i.e. $d_{2,0}$. This result has also been proved by employing a more formal approach in [14] and the expression of $d_{2,0}$ is given there. Consequently, it can be shown that $P_{m}(d)$ has a unique nonnegative root $d_{m, 0}$ such that $d_{m, 0}=\max _{k}\left\{d_{m, k}\right\}, k \in$ $\{0, m\}$, and $0 \leq d_{m, 0} \leq d_{m-1,0} \leq \ldots \leq d_{2,0} \leq d_{1,0}$ by extending the same reasoning for larger $m$ values [16]. Finally, $d_{0}=d_{m, 0}$ must be set in (9) for evaluating $\mu_{C(\widetilde{\mathbf{H}})}, \sigma_{C(\widetilde{\mathbf{H}})}^{2}$ and eventually $P_{\text {out }}$. For $m=3$, the roots of $P_{3}(d)$ can be expressed as

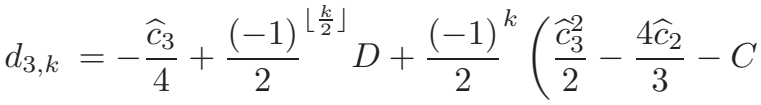

$$
\begin{aligned}
& \left.+(-1)^{\left(\left\lfloor\frac{k}{2}\right\rfloor+1\right)} \frac{\widehat{c}_{3}^{3}-4 \widehat{c}_{3} \widehat{c}_{2}+8 \widehat{c}_{1}}{4 D}\right)^{\frac{1}{2}}
\end{aligned}
$$

where $D=\sqrt{\frac{\widehat{c}_{3}^{2}}{4}-\frac{2 \widehat{c}_{2}}{3}+C}, C=\frac{2^{\frac{1}{3}} B}{3\left(A+\sqrt{-4 B^{3}+A^{2}}\right)^{\frac{1}{3}}}+$ $\left(\frac{A+\sqrt{-4 B^{3}+A^{2}}}{54}\right)^{\frac{1}{3}}, B=\widehat{c}_{2}^{2}-3 \widehat{c}_{3} \widehat{c}_{1}+12 \widehat{c}_{0}, A=2 \widehat{c}_{2}^{3}-9 \widehat{c}_{3} \widehat{c}_{2} \widehat{c}_{1}+$ $27 \widehat{c}_{1}^{2}+27 \widehat{c}_{3}^{2} \widehat{c}_{0}-72 \widehat{c}_{2} \widehat{c}_{0}, \widehat{c}_{i}=c_{m, i} / c_{m, 4}$ for $i \in\{0,3\}$, and $c_{m, i}$ are the coefficients of $P_{m}(d)=\sum_{i=0}^{m+1} c_{m, i} d^{i}$. In the case of $m=4$, the roots of $P_{4}(d)$ are given in [17], whereas the function "solve" in Matlab can be used to obtain $d_{m, k}$ for $m \geq 5$.

\section{NumericAl RESUlTS AND APPLICATIONS}

Our closed-form approximation in (10) can be used to accurately evaluate and compare the outage probability of DMIMO system faster than Monte-Carlo (MC) simulations, e.g. 10,000 times faster when $10^{6}$ channel realizations are used in MC. Its accuracy is discussed in the next section.

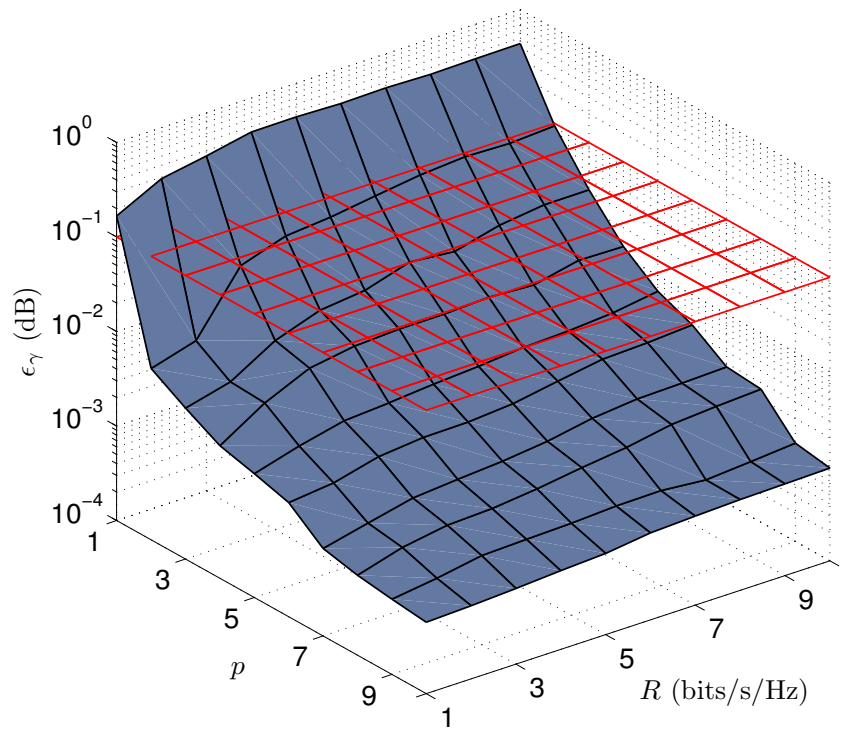

Fig. 3. Accuracy of our closed-form approximation in (10) against the number of antennas $p$ and rate $R$ for a 2-BSs DMIMO system and $\Delta_{\gamma}=5$ dB.

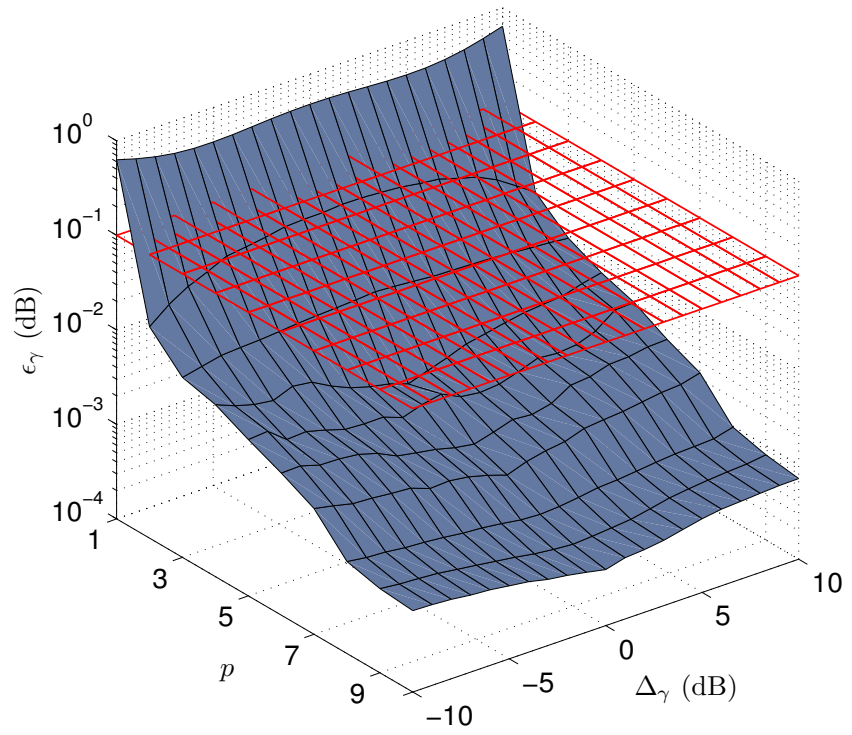

Fig. 4. Accuracy of our closed-form approximation in (10) against the number of antennas $p$ and SNR offset $\Delta_{\gamma}$ for a 2-BSs DMIMO system and $R=4$.

\section{A. Accuracy of our closed-form approximation in (10)}

The accuracy of our closed-form approximation is first displayed in Fig. 2, where the outage probability which has been computed by using (10), i.e. $P_{\mathrm{AN}}$, is compared against the outage probability that has been obtained via MC simulation by considering $10^{6}$ channel realizations, i.e. $P_{\mathrm{MC}}$, as a function of the rate $R$ and for various SNR values, numbers of nodes and transmit antennas. The graph shows a tight fitness between $P_{\mathrm{AN}}$ and $P_{\mathrm{MC}}$ for the various considered settings and, hence, it indicates graphically the accuracy of our proposed closed-form approximation in (10).

In order to quantify the accuracy of (10) and to assess its dependency to the values of $\gamma, \gamma_{i}, m, p, q, R$, we use the metric $\varepsilon_{\gamma}(\mathrm{dB}) \triangleq\left|\gamma_{\mathrm{AN}}(\mathrm{dB})-\gamma_{\mathrm{MC}}(\mathrm{dB})\right|$. It provides the absolute 


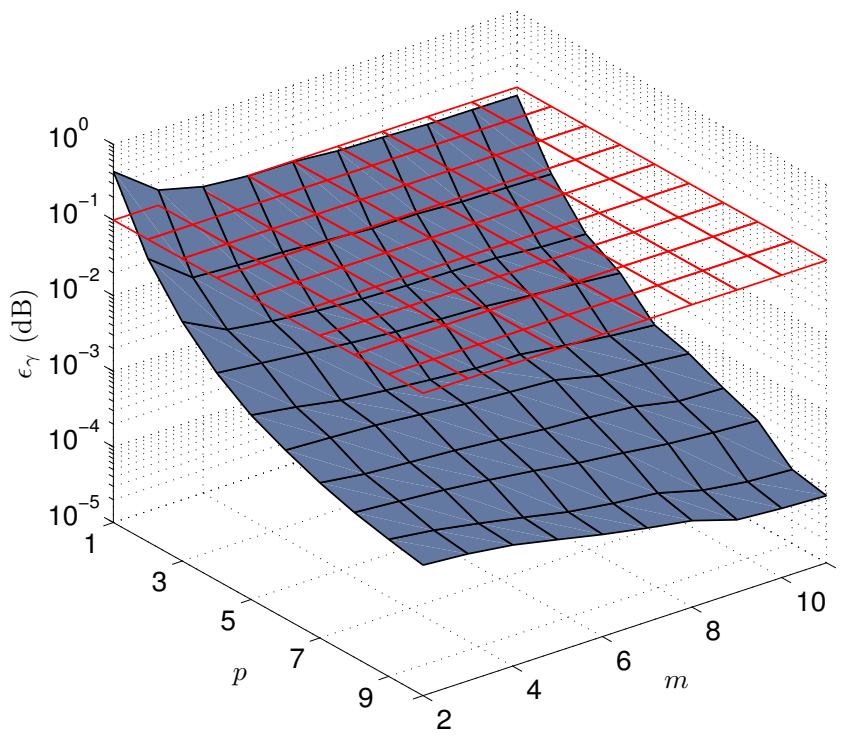

Fig. 5. Accuracy of our closed-form approximation in (10) against the number of antennas $p$ and number of nodes $m$ for a $m$-BSs DMIMO system and $R=4$.

difference in $\mathrm{dB}$ between the analytical SNR $\gamma_{\mathrm{AN}}$ and the SNR obtained via MC simulation $\gamma_{\mathrm{MC}}$ that are required to achieve a certain rate $R$ for a given outage probability $P_{\text {out }}$. The lower is $\varepsilon_{\gamma}(\mathrm{dB})$, the more accurate is (10). In Fig. 3 the metric $\varepsilon_{\gamma}(\mathrm{dB})$ is plotted as a function of $p$ and $R$ with $\Delta_{\gamma}=5 \mathrm{~dB}$ for $P_{\text {out }}=$ $0.01, \gamma=0 \mathrm{~dB}, m=2, \beta=1$, i.e. $p=q$, and considering the downlink case. Notice that $\Delta_{\gamma}(\mathrm{dB}) \triangleq \gamma_{2}(\mathrm{~dB})-\gamma_{1}(\mathrm{~dB})$ is the SNR offset between the two BSs. This graph clearly points out that the accuracy of (10) increases as the number of antennas $p$ increases, for any rate $R$. In Fig. $4, \varepsilon_{\gamma}(\mathrm{dB})$ is plotted as a function of $p$ and $\Delta_{\gamma}$ with $R=4$ and the same other settings as in Fig. 3. The results show that (10) is more accurate for lower $\Delta_{\gamma}$ values and confirm the increase of accuracy for larger numbers of antennas $p$. Finally in Fig. 5, $\varepsilon_{\gamma}(\mathrm{dB})$ is plotted as a function of $p$ and $m$ with $R=4$ for $P_{\text {out }}=0.01$, $\gamma=0 \mathrm{~dB}, \gamma_{i}=5\left(i-1-\left\lfloor\frac{m-1}{2}\right\rfloor\right), \beta=1$, i.e. $p=q$, and considering the downlink case. The graph indicates that the accuracy of (10) also increases with the number of nodes $m$ but at a lower rate than with the number of antennas $p$. Overall, we can notice that a sufficient accuracy, i.e. $\varepsilon_{\gamma}<0.1 \mathrm{~dB}$, is reached for $p \geq 2$ regardless of $m, R$ and $\Delta_{\gamma}$. Notice that similar results have been obtained for the uplink case.

\section{B. Other Applications}

The theoretical throughput of communication system can be expressed as $T=\left(1-P_{\text {out }}\right) R$ when an unlimited backhaul between each BS and the central unit is assumed. An upper bound on the achievable throughput for DMIMO system can then be easily obtained by using (10) in the previous equation. For instance, we plot in Fig. 6 the difference $\Delta_{T}$ between the theoretical throughput of MIMO and 2-BSs DMIMO systems as a function of the rate $R$ and SNR $\gamma_{2}(\mathrm{~dB})$ for the downlink case when $p=q=4$ and $\gamma=1$. Moreover, we fixed $\gamma_{1}=1$ for the DMIMO system and we considered an aggregate SNR $\gamma_{2}+1$ for the MIMO system, as it is indicated in the top right

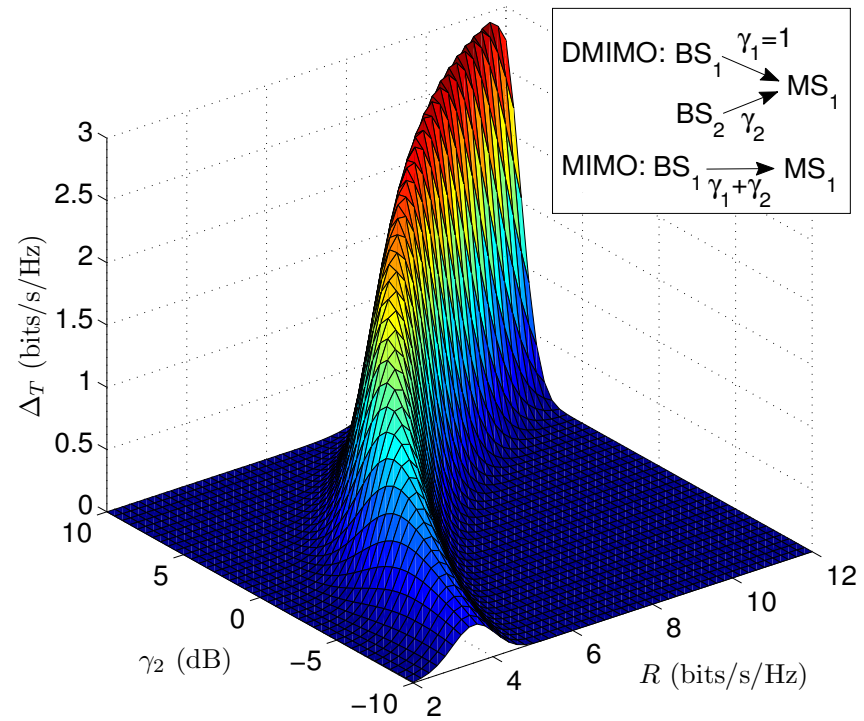

Fig. 6. Theoretical throughput comparison between MIMO and 2-BSs DMIMO systems against the rate $R$ and SNR $\gamma_{2}(\mathrm{~dB})$.

corner of Fig. 6, in order to fairly compare the two systems for the same total amount of power. Results in Fig. 6 show that a throughput gain can be achieved by using a DMIMO system instead of a MIMO system. However, this gain is quite localized; if the SNR is low, high rate cannot be supported and, hence, both systems are in outage, i.e. $P_{\text {out }}=1$ and $T=0$ for both systems (null plan on the right side of curve). For low rate and high SNR, both systems are saturated and achieved the same throughput (null plan on the left side of curve). Since, the DMIMO system saturates faster than the MIMO system by taking advantage of the spatial diversity, a gain is obtained in-between the two null regions. However, it should be mentioned that DMIMO system will induce extra costs in terms of backhaul load and signal processing as $m$ increases.

The expression $\mu_{C(\widetilde{\mathbf{H}})}$ in (9) can be used to evaluate $\mathrm{E}_{\mathbf{H}}\{C(\mathbf{H})\}$ for any MIMO communication systems where the MI can be expressed as $C(\mathbf{H})=\lambda \ln \left|\mathbf{I}+\mathbf{H} \boldsymbol{\Delta} \mathbf{H}^{\dagger}\right|$ with $\Delta=\operatorname{diag}\left(\left[\delta_{1}, \delta_{2}, \ldots, \delta_{N_{t}}\right]\right)$ being a $N_{t} \times N_{t}$ diagonal matrix, i.e. the MI of a MIMO system over a semicorrelated Rayleigh fading channel. Especially, in the case where $N_{r}>N_{t}$, which cannot be evaluated with the computable formula in [11] and is likely to happen in the DMIMO uplink. In the context of cooperative communication [18], we designed a novel amplifyand-forward scheme in [19], which outperforms any other existing schemes when only receive knowledge is available at the relay node, by using our result in (9). In addition, (10) can be used for evaluating the outage probability of the cooperative relay channel, i.e. when both source and relay nodes transmit to the destination node.

Following a similar approach as in [10], the mean and variance of the MI in (9) can be used to analyze the spectral efficiency of DMIMO cellular system with a similar accuracy compared to [10] but over a wider range of SNRs, since our approximations in (9) has been derived without considering any SNR restrictions. 


\section{CONCLUSION}

In this paper, the MI of DMIMO system has been proved to be asymptotically equivalent to a Gaussian RV and its corresponding mean and variance have been derived. Both these terms are expressed as a function of a root of a polynomial with degree $m+1$. Consequently, a proof of the uniqueness of the selection of the root for any $m$ values has been given. Then, a closed-form approximation of the outage probability for DMIMO system has been derived for both downlink and uplink scenarios by employing the mean and variance of the MI. Numerical results have shown the accuracy of our approximation for even low values of $m, p$ and $q$, and have indicated that the accuracy increases mainly with the number of nodes and antennas of the system. In addition, several applications for our analysis have been suggested: computation of theoretical throughput, design of cooperative communication scheme, evaluation of the cooperative relay channel outage probability and analysis of the spectral efficiency of DMIMO system.

\section{APPENDIX}

\section{DERIVATION INSIGHTS:}

\section{A. From equation (2) to (3)}

Let us re-express $\left|\mathbf{I}_{N_{r}}+\frac{\gamma}{n} \widetilde{\mathbf{H}} \widetilde{\mathbf{H}}^{\dagger}\right|$ in (2), as follows

$$
\phi(\widetilde{\mathbf{H}})=\left|\mathbf{I}_{N_{r}}+\frac{\gamma}{n} \widetilde{\mathbf{H}} \widetilde{\mathbf{H}}^{\dagger}\right|=\left|\begin{array}{cc}
\mathbf{I}_{N_{r}} & j \sqrt{\gamma / n} \widetilde{\mathbf{H}} \\
j \sqrt{\gamma / n} \widetilde{\mathbf{H}}^{\dagger} & \mathbf{I}_{N_{t}}
\end{array}\right| .
$$

Knowing that if the real part of all the eigenvalues of a complex matrix $\mathbf{A} \in \mathbb{C}^{N_{r} \times N_{r}}$ is positive then $|\mathbf{A}|^{-1}=$ $\int_{\mathbb{C}^{N_{r}}} e^{-\pi \mathbf{x}^{\dagger} \mathbf{A x}} d \mathbf{x}$ [20], (22) can be modified as

$$
\phi(\widetilde{\mathbf{H}})^{-1}=\int_{\substack{\mathbb{C}^{N_{r}}\\}} \int_{\mathbb{C}^{N_{t}}} e^{(-\pi[\mathbf{x} d \mathbf{y},}
$$

where $\mathbf{x} \in \mathbb{C}^{N_{r} \times 1}$ and $\mathbf{y} \in \mathbb{C}^{N_{t} \times 1}$. At this stage, we obtain by applying the replica method [15] and expanding (23) the following equation

$$
\begin{aligned}
\phi(\tilde{\mathbf{H}})^{-u} & =\prod_{a=1}^{u} \int_{\mathbb{C}^{N_{r}}} \int_{\mathbb{C}^{N_{t}}} e^{-\pi\left(\mathbf{x}_{a}^{\dagger} \mathbf{x}_{a}+\mathbf{y}_{a}^{\dagger} \mathbf{y}_{a}\right)} \\
& \times e^{-j \pi \sqrt{\gamma / n}\left(\mathbf{x}_{a}^{\dagger} \widetilde{\mathbf{H}} \mathbf{y}_{a}+\mathbf{y}_{a}^{\dagger} \widetilde{\mathbf{H}}^{\dagger} \mathbf{x}_{a}\right)} d \mathbf{x}_{a} d \mathbf{y}_{a} \\
& =\int_{\mathbb{C}^{N_{r} \times u}} \int_{\mathbb{C}^{N_{t} \times u}} e^{-\pi \operatorname{tr}\left[\mathbf{X}^{\dagger} \mathbf{X}+\mathbf{Y}^{\dagger} \mathbf{Y}\right]} \\
& \times e^{-j \pi \sqrt{\gamma / n} \operatorname{tr}\left[\widetilde{\mathbf{H}} \mathbf{Y} \mathbf{X}^{\dagger}+\mathbf{X} \mathbf{Y}^{\dagger} \widetilde{\mathbf{H}}^{\dagger}\right]} d \mathbf{X} d \mathbf{Y},
\end{aligned}
$$

where $u$ is a dummy variable used in the replica method, $\mathbf{X}=$ $\left[\mathbf{x}_{1}, \mathbf{x}_{2}, \ldots, \mathbf{x}_{u}\right]$ and $\mathbf{Y}=\left[\mathbf{y}_{1}, \mathbf{y}_{2}, \ldots, \mathbf{y}_{u}\right]$. Equation (3) is then obtained by averaging (24) with respect to $\mathbf{H}$.

\section{B. From equation (3) to (4)}

The integrand in (3) being bounded and continuous in all its variables, we can start by integrating (3) with respect to
$\mathbf{H}$, as follows

$$
\begin{aligned}
A & =\int_{\mathbb{C}^{N_{r} \times N_{t}}} \pi^{-N_{r} N_{t}} \psi(\mathbf{H}) d \mathbf{H} \\
& =\prod_{r=1}^{N_{r}} \prod_{t=1}^{N_{t}} \pi^{-1} \int_{\mathbb{C}} \exp \left\{-\left[j\left(\Re \mathbf{H}_{r, t}+j \Im \mathbf{H}_{r, t}\right)\left(\Re \boldsymbol{\Sigma}_{r, t}+j \Im \boldsymbol{\Sigma}_{r, t}\right)\right.\right. \\
& \times\left(\Re \mathbf{B}_{r, t}-j \Im \mathbf{B}_{r, t}\right)+j\left(\Re \mathbf{B}_{r, t}+j \Im \mathbf{B}_{r, t}\right)\left(\Re \boldsymbol{\Sigma}_{r, t}-j \Im \boldsymbol{\Sigma}_{r, t}\right) \\
& \left.\left.\times\left(\Re \mathbf{H}_{r, t}-j \Im \mathbf{H}_{r, t}\right)+\left(\Re\left(\mathbf{H}_{r, t}\right)^{2}+\left(\Im \mathbf{H}_{r, t}\right)^{2}\right)\right]\right\} d \mathbf{H}_{r, t}, \\
& =\prod_{r=1}^{N_{r}} \prod_{t=1}^{N_{t}} \pi^{-1} \int_{\mathbb{C}} \exp \left\{-\left[\left\{\Re \mathbf{H}_{r, t}+j\left(\Re \boldsymbol{\Sigma}_{r, t} \Re \mathbf{B}_{r, t}+\Im \boldsymbol{\Sigma}_{r, t}\right.\right.\right.\right. \\
& \left.\left.\times \Im \mathbf{B}_{r, t}\right)\right\}^{2}+\left(\Re \boldsymbol{\Sigma}_{r, t} \Re \mathbf{B}_{r, t}+\Im \boldsymbol{\Sigma}_{r, t} \Im \mathbf{B}_{r, t}\right)^{2}+\left\{\Im \mathbf{H}_{r, t}\right. \\
& \left.+j\left(\Re \boldsymbol{\Sigma}_{r, t} \Im \mathbf{B}_{r, t}+\Im \boldsymbol{\Sigma}_{r, t} \Re \mathbf{B}_{r, t}\right)\right\}^{2}+\left(\Re \boldsymbol{\Sigma}_{r, t} \Im \mathbf{B}_{r, t}+\Im \boldsymbol{\Sigma}_{r, t}\right. \\
& \left.\left.\left.\times \Re \mathbf{B}_{r, t}\right)^{2}\right]\right\} d \mathbf{H}_{r, t .} . \\
& =\prod_{r=1}^{N_{r}} \prod_{t=1}^{N_{t}} \exp -\left[\left(\Re \boldsymbol{\Sigma}_{r, t}^{2}+\Im \boldsymbol{\Sigma}_{r, t}^{2}\right)\left(\Re \mathbf{B}_{r, t}^{2}+\Im \mathbf{B}_{r, t}^{2}\right)\right] \\
& =\prod_{r=1}^{N_{r}} \prod_{t=1}^{N_{t}} \exp \left[-\left|\boldsymbol{\Sigma}_{r, t}\right|^{2}\left|\mathbf{B}_{r, t}\right|^{2}\right] \\
& =\exp \left(-\pi^{2} \frac{\gamma}{n} \operatorname{tr}\left[\left(\boldsymbol{\Sigma} \odot \mathbf{X Y}^{\dagger}\right)\left(\boldsymbol{\Sigma} \odot \mathbf{X Y}^{\dagger}\right)^{\dagger}\right]\right) .
\end{aligned}
$$

\section{From equation (5) to (6)}

Knowing that $\frac{1}{j 2 \pi} \int_{\mathcal{D}_{f_{0}}^{j}} \int_{\mathcal{D}_{g_{0}}} \exp \{f(-\kappa g+x)+g y\} d f d g=$ $\exp (x y / \kappa) / \kappa$ holds for any $x, y, \kappa, f, g \in \mathbb{C}$ [7], we can reexpress $\exp \left(-\operatorname{tr}\left[\pi^{2} \frac{\gamma}{n} \mathbf{W}^{\dagger} \mathbf{W} \mathbf{Y}^{\dagger} \mathbf{Y}\right]\right)$ in (5), as follows

$$
\begin{aligned}
A & =e^{\operatorname{tr}\left[-\pi^{2} \frac{\gamma}{n} \mathbf{W}^{\dagger} \mathbf{W} \mathbf{Y}^{\dagger} \mathbf{Y}\right]}=\prod_{a=1}^{u} \prod_{b=1}^{u} e^{-\pi^{2} \frac{\gamma}{n}\left(\mathbf{W}^{\dagger} \mathbf{W}\right)_{a, b}\left(\mathbf{Y}^{\dagger} \mathbf{Y}\right)_{b, a}} \\
& =\prod_{a=1}^{u} \prod_{b=1}^{u} \frac{n}{j 2 \pi} \int_{\mathcal{D}_{\mathbf{D}_{b, a}}^{j}} \int_{\mathcal{D}_{\mathbf{G}_{a, b}}} e^{\mathbf{D}_{b, a}\left(n \mathbf{G}_{a, b}-\pi \sqrt{\gamma}\left(\mathbf{W}^{\dagger} \mathbf{W}\right)_{a, b}\right)} \\
& \times e^{-\pi \sqrt{\gamma} \mathbf{G}_{a, b}\left(\mathbf{Y}^{\dagger} \mathbf{Y}\right)_{b, a}} d \mathbf{D}_{b, a} d \mathbf{G}_{a, b} \\
& =\left(\frac{n}{j 2 \pi}\right)^{u^{2}} \int_{\mathcal{D}_{d}^{j}} \int_{\mathcal{D}_{g}} e^{\operatorname{tr}\left[n \mathbf{D G}-\pi \sqrt{\gamma}\left(\mathbf{D} \mathbf{W}^{\dagger} \mathbf{W}+\mathbf{G} \mathbf{Y}^{\dagger} \mathbf{Y}\right)\right]} d \mathbf{D} d \mathbf{G} .
\end{aligned}
$$

Inserting (25) into (5) and followed by straightforward simplifications, we obtain

$$
\begin{aligned}
M_{C(\widetilde{\mathbf{H}})}(-u / \lambda) & =\left(\frac{n}{j 2 \pi}\right)^{u^{2}} \int_{\mathcal{D}_{d}^{j}} \int_{\mathcal{D}_{g}} e^{n \operatorname{tr}[\mathbf{D G}]} \\
& \times \int_{\mathbb{C}^{N_{r} \times u}} e^{-\pi \operatorname{tr}\left[\mathbf{X}^{\dagger} \mathbf{X}+\sqrt{\gamma} \mathbf{D} \mathbf{W}^{\dagger} \mathbf{W}\right]} d \mathbf{X} \\
& \times \int_{\mathbb{C}^{N_{t} \times u}} e^{-\pi \operatorname{tr}\left[\mathbf{Y}^{\dagger} \mathbf{Y}\left(\mathbf{I}_{u}+\sqrt{\gamma} \mathbf{G}\right)\right]} d \mathbf{Y} d \mathbf{D} d \mathbf{G}
\end{aligned}
$$

Notice that $\operatorname{tr}\left[\mathbf{Y}^{\dagger} \mathbf{Y}\left(\mathbf{I}_{u}+\sqrt{\gamma} \mathbf{G}\right)\right]=\sum_{i=1}^{N_{t}} \mathbf{Y}_{i}\left(\mathbf{I}_{u}+\sqrt{\gamma} \mathbf{G}\right) \mathbf{Y}_{i}^{\dagger}$ and $\operatorname{tr}\left[\mathbf{X}^{\dagger} \mathbf{X}+\sqrt{\gamma} \mathbf{D} \mathbf{W}^{\dagger} \mathbf{W}\right]=\sum_{i=1}^{N_{r}} \mathbf{X}_{i}\left(\mathbf{I}_{u}+\sqrt{\gamma} \gamma_{\lfloor i / p\rfloor} \mathbf{D}\right) \mathbf{X}_{i}^{\dagger}$ in (26), where $\mathbf{X}_{i}$ and $\mathbf{Y}_{i}$ are the $i$-th row vectors of $\mathbf{X}$ and $\mathbf{Y}$, respectively, and $\lfloor$.$\rfloor is the integer floor operator. Then,$ $M_{C(\tilde{\mathbf{H}})}(-u / \lambda)$ can be formulated as in (6) by using $|\mathbf{A}|^{-1}=$ $\int_{\mathbb{C}^{N_{r}}} e^{-\pi \mathbf{x}^{\dagger} \mathbf{A} \mathbf{x}} d \mathbf{x}$ for integrating the integrand with respect to $\mathbf{X}$ and $\mathbf{Y}$ in (26). 


\section{From equation (6) to (7)}

In order to asymptotically compute the integral in (6), i.e. for $m p, q \rightarrow \infty$, we apply the multidimensional saddle point integration method [13]. First, we expand the Taylor series of $\varphi(\mathbf{D}, \mathbf{G})$ for $\mathbf{D}=\mathbf{D}_{0}+\delta \mathbf{D}$ and $\mathbf{G}=\mathbf{G}_{0}+\delta \mathbf{G}$ to its second order, and we obtain

$\varphi(\mathbf{D}, \mathbf{G}) \approx \varphi\left(\mathbf{D}_{0}, \mathbf{G}_{0}\right)+\operatorname{tr}\left[\left(\mathbf{G}_{\mathbf{0}}-\alpha \sum_{i=1}^{m}\left(\omega v_{i} \mathbf{I}_{u}+\mathbf{D}_{\mathbf{0}}\right)^{-1}\right) \delta \mathbf{D}\right.$

$+\left(\mathbf{D}_{\mathbf{0}}-\beta\left(\omega \mathbf{I}_{u}+\mathbf{G}_{\mathbf{0}}\right)^{-1}\right) \delta \mathbf{G}+\frac{1}{2}\left\{\alpha \sum_{i=1}^{m}\left(\omega v_{i} \mathbf{I}_{u}+\mathbf{D}_{\mathbf{0}}\right)^{-2}\right.$

$\left.\left.\times(\delta \mathbf{D})^{2}+2 \delta \mathbf{D} \delta \mathbf{G}+\beta\left(\omega \mathbf{I}_{u}+\mathbf{G}_{\mathbf{0}}\right)^{-2}(\delta \mathbf{G})^{2}\right\}\right]$.

According to (27), we obtain two critical points as follows

$$
\mathbf{G}_{0}=\alpha \sum_{i=1}^{m}\left(\omega v_{i} \mathbf{I}_{u}+\mathbf{D}_{0}\right)^{-1} \text { and } \mathbf{D}_{0}=\beta\left(\omega \mathbf{I}_{u}+\mathbf{G}_{0}\right)^{-1} \text {. }
$$

The matrix $\mathbf{D}_{0}$ is invariant in replica space and is thus proportional to the identity matrix $\mathbf{I}_{u}$ [5]. Consequently, we set $\mathbf{D}_{0}=d \mathbf{I}_{u}$ and assume that the absolute maximum of $\Re \varphi(\mathbf{D}, \mathbf{G})$ occurs for $\Re \varphi\left(\mathbf{D}_{0}, \mathbf{G}_{0}\right)$. Inserting the first equation into the second equation of (28) and considering that $\mathbf{D}_{0}=$ $d \mathbf{I}_{u}$, we obtain the polynomial equation in (8). We select one root amongst the $m+1$ roots of $P_{m}(d)$ in (8) and denote it $d_{0}$. We then set $\mathbf{D}_{0}=d_{0} \mathbf{I}_{u}$ and get $\mathbf{G}_{0}$ by using the first equation of (28). Next, we obtain

$$
\varphi(\mathbf{D}, \mathbf{G}) \approx \varphi\left(\mathbf{D}_{0}, \mathbf{G}_{0}\right)+\frac{1}{2} \operatorname{tr}\left[\widehat{d_{0}}(\delta \mathbf{D})^{2}+2 \delta \mathbf{D} \delta \mathbf{G}+\widehat{g_{0}}(\delta \mathbf{G})^{2}\right]
$$

by applying $\mathbf{D}_{0}$ and $\mathbf{G}_{0}$ in (27). Inserting (29) into (6) and integrating $\int_{\mathcal{D}_{d}^{j}} \int_{\mathcal{D}_{g}} \exp \left(\frac{n}{2} \operatorname{tr}\left[\widehat{d_{0}}(\delta \mathbf{D})^{2}+2 \delta \mathbf{D} \delta \mathbf{G}+\widehat{g_{0}}(\delta \mathbf{G})^{2}\right]\right)$ $\times d(\delta \mathbf{D}) d(\delta \mathbf{G})$ along the steepest descent paths for $\delta \mathbf{D}$ and $\delta \mathbf{G}$, we finally obtain the expression in (7).

\section{E. Equation (11)}

Let $\sigma_{Z}^{2} \in \mathbb{R}_{+}^{\star}$, i.e. $0<-\lambda^{2} \ln \left(1-\frac{\alpha}{\beta} \sum_{i=1}^{m}\left(\frac{d_{0}}{d_{0}+\omega v_{i}}\right)^{2}\right)$ $<+\infty$ with (7), it implies that

$$
\begin{aligned}
\sigma_{Z}^{2} \in \mathbb{R}_{+}^{\star} & \Leftrightarrow 1>1-\frac{\alpha}{\beta} \sum_{i=1}^{m}\left(\frac{d_{0}}{d_{0}+\omega v_{i}}\right)^{2}>0, \\
& \Leftrightarrow 0<\sum_{i=1}^{m}\left(\frac{d_{0}}{d_{0}+\omega v_{i}}\right)^{2}<\frac{q}{p},
\end{aligned}
$$

knowing that $\beta / \alpha=q / p$. Moreover, the inequality $\left(\frac{d_{0}}{d_{0}+\omega \hat{v}}\right)^{2} \leq\left(\frac{d_{0}}{d_{0}+\omega v_{i}}\right)^{2}$ holds for any $i \in\{1, m\}$, with $\widehat{v}=\max _{i}\left\{v_{i}\right\}$. Thus, we can write

$$
\begin{aligned}
& m\left(\frac{d_{0}}{d_{0}+\omega \widehat{v}}\right)^{2} \leq \sum_{i=1}^{m}\left(\frac{d_{0}}{d_{0}+\omega v_{i}}\right)^{2}<\frac{q}{p} \\
& \Leftrightarrow m\left(1-\frac{\omega \widehat{v}\left(2 d_{0}+\omega \widehat{v}\right)}{\left(d_{0}+\omega \widehat{v}\right)^{2}}\right)<\frac{q}{p} \\
& \Leftrightarrow Q\left(d_{0}\right)=d_{0}^{2}+2\left(\frac{q}{q-p m}\right) \omega \widehat{v} d_{0}+\left(\frac{q}{q-p m}\right) \omega^{2} \widehat{v}^{2}>0 .
\end{aligned}
$$

The two roots of the polynomial $Q\left(d_{0}\right)$ are then given by

$$
d_{\mathrm{a}}=\omega \widehat{v}\left[\frac{q}{p m-q}+\frac{\sqrt{p m q}}{|p m-q|}\right] \text { and }
$$

$$
d_{\mathrm{b}}=\omega \widehat{v}\left[\frac{q}{p m-q}-\frac{\sqrt{p m q}}{|p m-q|}\right],
$$

respectively, where $d_{\mathrm{b}} \leq d_{\mathrm{a}}$. In the case that $q=p m$, then $\omega \widehat{v}\left(2 d_{0}+\omega \widehat{v}\right)>0$ in the third line of (31) and, hence, $d_{0}>$ $-\omega \widehat{v} / 2$. In the case that $q>p m$ then $\left(\frac{q}{p m}-1\right)>0$ and, hence, $Q\left(d_{0}\right)$ is positive outside the interval $\left[d_{\mathrm{b}}, d_{\mathrm{a}}\right]$. In the case that $q<p m$ then $\left(\frac{q}{p m}-1\right)<0$ and, hence, $Q\left(d_{0}\right)$ is positive inside the interval $\left[d_{\mathrm{b}}, d_{\mathrm{a}}\right]$.

\section{ACKNOWLEDGMENT}

The first author wish to thank his beloved wife for her support and helpful comments in preparing this paper.

\section{REFERENCES}

[1] G. J. Foschini and M. J. Gans, "On Limits of Wireless Communications in a Fading Environment when using Multiple Antennas," Wireless Personal Commun., vol. 6, pp. 311-335, 1998.

[2] I. E. Telatar, "Capacity of Multi-Antenna Gaussian Channels," Europ. Trans. Telecommun. and Related Technol., vol. 10, no. 6, pp. 585-596, Nov. 1999.

[3] M. K. Simon and M.-S. Alouini, Digital Communication over Fading Channels: A Unified Approach to Performance Analysis. ed. Wiley, Feb. 2000.

[4] A. M. Sengupta and P. P. Mitra, "Capacity of Multivariate Channels with Multiplicative Noise: I.Random Matrix Techniques and Large- $N$ Expansions for Full Transfer Matrices," LANL arXiv:physics, Oct. 2000.

[5] A. L. Moustakas, S. H. Simon, and A. M. Sengupta, "MIMO Capacity through Correlated Channels in the Presence of Correlated Interferers and Noise: A (not so) Large $N$ Analysis," IEEE Trans. Inform. Theory, vol. 49, no. 10 , pp. 2545-2561, Oct. 2003.

[6] B. H. Hochwald, T. L. Marzetta, and V. Tarokh, "Multi-Antenna Channel Hardening and its Implications for Rate Feedback and Schedulling," IEEE Trans. Inform. Theory, vol. 50, no. 9, pp. 1893-1909, Sept. 2004.

[7] E. Biglieri and G. Taricco, Transmission and Reception with Multiple Antennas: Theoretical Foundations. Now Publishers Inc., 2004.

[8] P. J. Smith and M. Shafi, "On a Gaussian Approximation to the Capacity of Wireless MIMO Systems,' in Proc. IEEE ICC '02, New-York, USA, Apr. 2002, pp. 406-410.

[9] W. Roh and A. Paulraj, "Outage Performance of the Distributed Antenna Systems in a Composite Fading Channel," in Proc. IEEE VTC 2002-Fall, vol. 3, Sept. 2002, pp. 1520- 1524.

[10] D. Wang, X. You, J. Wang, Y. Wang, and X. Hou, "Spectral Efficiency of Distributed MIMO Cellular Systems in a Composite Fading Channel," in Proc. IEEE ICC '08, May 2008, pp. 1259- 1264.

[11] P. J. Smith, S. Roy, and M. Shafi, "Capacity of MIMO Systems with Semicorrelated Flat Fading," IEEE Trans. Inform. Theory, vol. 49, no. 10, pp. 2781-2787, Oct. 2003.

[12] M. L. Mehta, Random Matrices. Academic Press, New York, 1991.

[13] N. Bleistein and R. A. Handelsman, Asymptotic Expensions of Integrals. Dover, 1986.

[14] F. Héliot, R. Hoshyar, and R. Tafazolli, "A Closed-Form Approximation of the Outage Probability for Distributed MIMO Systems," in Proc. IEEE SPAWC 2009, Perugia, Italy, June 2009.

[15] S. F. Edwards and P. Anderson, "Theory of Spin Glasses," J. Phys. F:Metal Phys., vol. 5, p. 965974, May 1975.

[16] F. Héliot, R. Hoshyar, and R. Tafazolli, "An Accurate ClosedForm Approximation of the Distributed MIMO Outage Probability: Derivation Insights," Tech. Rep., July 2010. [Online]. Available: http://membres.multimania.fr/fheliot/pub/Technote2.pdf

[17] "Wolfram Mathworld Website." [Online]. Available: http://mathworld. wolfram.com/QuinticEquation.html

[18] A. Sendonaris, E. Erkip, and B. Aazhang, "User Cooperation Diversity Part I \& II- System Description / Implementation Aspects and Performance Analysis," IEEE Trans. Commun., vol. 51, no. 11, pp. 1927-1948, Nov. 2003.

[19] F. Héliot, S. Fazel, R. Hoshyar, and R. Tafazolli, "Receive Knowledge Only Power Allocation for Nonregenerative Cooperative MIMO Communication," in Proc. IEEE SPAWC 2009, Perugia, Italy, June 2009.

[20] M. Elia and G. Taricco, "Integration of the Exponential Function of a Complex Quadratic Form," Applied Mathematics E-notes, vol. 3, pp. 95-98, 2003. 